\title{
Investigation of highly expressed PCSK9 in atherosclerotic plaques and ox-LDL-induced endothelial cell apoptosis
}

\author{
JIAO LI, XUE LIANG, YUANYUAN WANG, ZHAO XU and GUANGPING LI \\ Tianjin Key Laboratory of Ionic-Molecular Function of Cardiovascular Disease, Department of Cardiology, \\ Tianjin Institute of Cardiology, The Second Hospital of Tianjin Medical University, Tianjin 300211, P.R. China
}

Received March 3, 2016; Accepted February 2, 2017

DOI: $10.3892 / \mathrm{mmr} .2017 .6803$

\begin{abstract}
The present study aimed to explore the direct toxicity of proprotein convertase subtilisin/kexin type 9 (PCSK9) to atherosclerosis (AS) and its association with apoptotic endothelial cells. Apolipoprotein $\mathrm{E}^{-/-}$mice were randomly divided into two groups, control and experimental. The control group was administered a normal diet and the experimental group was administered a high-fat diet. After 20 weeks, the aorta was isolated and dissected. Hematoxylin and eosin staining, and immunohistochemical analysis were performed. Human umbilical vein endothelial cells were incubated with varied concentrations of oxidized low-density lipoprotein (ox-LDL) for different times. The apoptotic rate was detected by flow cytometry. Western blotting and reverse transcription-quantitative polymerase chain reaction analysis were conducted to detect the expression of PCSK9, B-cell lymphoma 2 (Bcl-2), bcl-2-like protein 4 (Bax) and caspase-3. Short hairpin (sh) RNA-PCSK9 was transfected into endothelial cells using lentiviral transfection. The expression levels of PCSK9, Bax, Bcl-2, caspase-3 and the mitogen-activated protein kinase (MAPK) pathway proteins were detected. The high-fat group was successfully established as an AS model and PCSK9 was highly expressed in the AS plaque. Treatment with ox-LDL induced apoptosis and increased mRNA and protein levels of PCSK9. PCSK9 mRNA and proteins levels were downregulated by shRNA-PCSK9. The deficiency of PCSK9 markedly inhibited the expression of pro-apoptotic proteins and promoted anti-apoptotic proteins. In addition, phosphorylation of p38 and c-Jun N-terminal kinases was altered by shRNA-PCSK9. Targeting of PCSK9 by shRNA-PCSK9 may
\end{abstract}

Correspondence to: Professor Guangping Li, Tianjin Key Laboratory of Ionic-Molecular Function of Cardiovascular Disease, Department of Cardiology, Tianjin Institute of Cardiology, The Second Hospital of Tianjin Medical University, 23 Pingjiang Road, Tianjin 300211, P.R. China

E-mail: tjcardiol@126.com

Key words: proprotein convertase subtilisin/kexin type 9, atherosclerosis, endothelial cells, apoptosis, mitogen-activated protein kinase signaling repress endothelial cell apoptosis through MAPK signaling in AS, providing a novel direction for understanding the mechanism and treatment of AS.

\section{Introduction}

Atherosclerosis (AS) is the leading cause of cardiovascular disease and is characterized by structural alterations in the vascular walls of medium and large arteries. A large number of studies $(1,2)$ have indicated that AS is caused by several factors; however, its exact pathogenesis remains unclear. Inflammatory reaction and lipid metabolism abnormality are two theories of AS (3). Although the development of AS is caused by the dysfunction of a number of pathways, one of the important risk factors is oxidized low-density lipoprotein (ox-LDL) causing endothelial dysfunction (4). In the development of AS, apoptosis of the endothelial cells is able to alter endothelial integrity and increase permeability, thus promoting damage of the blood vessels and the formation of a plaque. Among the numerous risk factors of AS, ox-LDL is the primary factor in the promotion of apoptosis in endothelial cells (5). Ox-LDL is able to accelerate the proliferation and migration of smooth muscle cells, monocytes, macrophages and fibroblasts, in addition to the oxidative stress response and cell damage of endothelial cells $(6,7)$, eventually leading to AS plaque formation.

Proprotein convertase subtilisin/kexin type 9 (PCSK9) is a member of the proprotein convertase subtilisin/kexin family, encoding neural apoptosis-regulated convertase 1 protein. PCSK9 is a newly identified gene, associated with familial autosomal dominant hypercholesterolemia (8). The inhibition of PCSK9 is able to significantly reduce the plasma low-density lipoprotein cholesterol (LDL-C) levels in the normal population and patients with high cholesterol taking statins $(9,10)$, reducing the incidence and mortality of cardiovascular disease (11). In the present study, the association between PCSK9 and hyperlipidemia was investigated. That is, whether PCSK9 can increase the levels of LDL-C in plasma by degrading the low-density lipoprotein receptor (LDLR) of the hepatocyte surface (12). Following this, PCSK9 regulates the metabolism of cholesterol and promotes the development of AS (13). However, there is has been no advancement in identifying the direct association between PCSK9 and AS, providing the context for the present study, which aims to 
investigate the direct association between PCSK9 and AS and its possible mechanism.

\section{Materials and methods}

Materials. The human umbilical vein endothelial cell (HUVEC) line EAhy926 was obtained from the Institute of Pharmacology, Medical University of Tianjin (Tianjin, China). Ox-LDL was purchased from Xinyuan Jiahe Biotechnology (Beijing, China). Basic Dulbecco's modified Eagle's medium (DMEM) and fetal bovine serum were purchased from Invitrogen (Thermo Fisher Scientific, Inc., Waltham, MA, USA). RNase inhibitor and Moloney murine leukemia virus reverse transcriptase was purchased from Takara Biotechnology Co., Ltd. (Dalian, China). Monoclonal antibodies against PCSK9, B-cell lymphoma 2 (Bcl-2), bcl-2-like protein 4 (Bax), caspase-3, p38, phosphorylated (p)-p38, extracellular signal-regulated kinases (ERK), p-ERK, c-Jun N-terminal kinases (JNK) and p-JNK were purchased from Abcam (Cambridge, MA, USA). SYBR Green PCR Premix was purchased from Biocentury TransGene (Beijing, China). Annexin V fluorescein isothiocyanate (FITC) apoptosis kit was purchased from BD Biosciences (Franklin Lakes, NJ, USA). The flow cytometer (version, BDFACS Verse) was purchased from BD Biosciences and FlowJo software version 7.6 (FlowJo, LLC, Ashland, OR, USA) was used. The lentiviral packaging system, containing helper plasmids and target plasmids, was purchased from CWBIO (Beijing, China).

Animal AS model. A total of 12 Apolipoprotein E (ApoE) $)^{-1-}$ mice on a $\mathrm{C} 57$ black 6 background were purchased from Beijing University (Beijing, China). Mice used in this study were male, 6-8 weeks old, weighed 20-25 g, and were housed in the Second Hospital of Tianjin Medical University Animal Care Facility under pathogen-free conditions, according to institutional guidelines (temperature, $22 \pm 2{ }^{\circ} \mathrm{C}$; relative

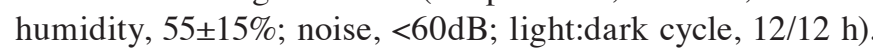
All animal study protocols were approved by the Animal Care and Utilization Committee of Tianjin Medical University. At 8 weeks of age, $\mathrm{ApoE}^{-/-}$mice were randomly divided into two groups ( $\mathrm{n}=6$ for each). Mice in the control group received a standard diet, while mice in the AS model group received a high-fat diet $(0.25 \%$ cholesterol and $15 \%$ cocoa butter $)$ to induce atherosclerotic plaques. After 20 weeks, the animals were sacrificed to obtain the aorta. The aorta was fixed in $4 \%$ paraformaldehyde overnight for immunohistochemical analysis and hematoxylin and eosin staining.

Cell culture. EAhy926 cells were grown in a monolayer and maintained in DMEM containing $10 \%$ fetal bovine serum, $100 \mathrm{U} / \mathrm{ml}$ penicillin $\mathrm{G}$ and $100 \mu \mathrm{g} / \mathrm{ml}$ streptomycin and cultured at $37^{\circ} \mathrm{C}$ in a humidified incubator with $5 \% \mathrm{CO}_{2}$. When the cells reached the platform stage, cell culture, cell cryopreservation and subsequent experiments were performed.

Detection of apoptosis rate. EAhy926 cells were seeded in a 24 -well plate $\left(2 \times 10^{5} /\right.$ well) and cultured with ox-LDL. The cells were collected at the end of each time point and stained by FITC Annexin V and propidium iodide (PI). Apoptosis rates were detected by flow cytometry in accordance with the manufacturer's protocols.
Lentiviral transfection. The small hairpin (sh)RNA sequence of PCSK9 was identified on the Sigma-Aldrich website (www.sigmaaldrich.com/life-science/functionalgenomics-and-rnai/shrna/individual-genes.html) and synthesized by GENEWIZ(Suzhou, China). The recombinant plasmid was added to the Escherichia coli DH5 $\alpha$ cells (CWBIO), according to the molecular cloning manual. The mixed liquid was evenly coated on the solid lysogeny broth (LB) medium plate and cultured $12-16 \mathrm{~h}$ at $37^{\circ} \mathrm{C}$ in a humidified incubator. The discrete white colonies were inoculated into $5 \mathrm{ml} \mathrm{LB}$ liquid culture medium containing 4-(aminomethyl) piperidine $(100 \mathrm{~g} / \mathrm{ml})$ then placed in a constant temperature oscillator overnight. The plasmid was extracted using the PurePlasmid Mini kit (CWBIO; Beijing, China). The lentiviral packaging system containing three helper plasmids (Rev $2.5 \mu \mathrm{g}$, VSVG $3 \mu \mathrm{g}$ and pMDL $5 \mu \mathrm{g}$ ) and 279-target plasmid (279-vector $12 \mu \mathrm{g}, 279$-iPCSK9-1 $12 \mu \mathrm{g}$ and 279-iPCSK9-2 $12 \mu \mathrm{g}$ ) were co-transfected into $293 \mathrm{~T}$ cells using polyethylenimine. The virus was collected and transfected into EAhy926 cells. shRNA-PCSK9 sequences are presented in Table I.

Isolation of RNA and reverse transcription-quantitative polymerase chain reaction $(R T-q P C R)$. Total RNA was extracted from cultured cells using TRIzol reagent (Invitrogen; Thermo Fisher Scientific, Inc.). Reverse transcription was performed using the M-MLV Reverse Transcription system (Takara Biotechnology Co., Ltd). RT-qPCR was performed using the SYBR-Green PCR kit as described by the manufacturer (Biocentury TransGen). Reference gene for the RT-qPCR was GAPDH. The thermocycling conditions were as follows: Denaturation $\left(95^{\circ} \mathrm{C} ; 30 \mathrm{sec}\right)$, annealing $\left(58^{\circ} \mathrm{C} ; 30 \mathrm{sec}\right)$, extension $\left(72^{\circ} \mathrm{C} ; 30 \mathrm{sec}\right)$. The qPCR reaction mix contained: $10 \mu \mathrm{l}$ 2X SYBR-Green PCR Premix, $1.5 \mu \mathrm{l}$ cDNA (dilution, 1:20),

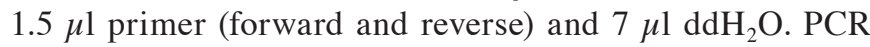
primers are listed in Table I. The qPCR reaction was carried out using an Applied Biosystems 7500 Fast Real-Time PCR System (Applied Biosystems; Thermo Fisher Scientific, Inc.). The relative amount of all mRNAs was calculated using the $2^{-\Delta \Delta \mathrm{Cq}}$ method (14).

Western blot analysis. The cells were collected at the end of each experiment, then washed three times with pre-cooled PBS. Proteins were extracted from the cells using radioimmunoprecipitation assay buffer (Beyotime Institute of Biotechnology, Haimen, China). The protein concentration was determined using the 2-D Quant kit (GE Healthcare Life Sciences, Chalfont, UK) method. The samples (30 $\mu \mathrm{g} / \mathrm{lane})$ were separated on a $10 \%$ SDS-PAGE, and then transferred onto a PVDF membrane. Subsequently, the membrane was blocked with $5 \%$ non-fat milk for $1 \mathrm{~h}$ at room temperature. The membrane was incubated with antibodies against $\beta$-actin, PCSK9, Bax, caspase-3, Bcl-2, p38, p-p38, ERK, p-ERK, JNK and p-JNK at $4^{\circ} \mathrm{C}$ overnight (Table II). Following the overnight incubation, the membrane was washed three times with TBS-Tween-20 (TBST) for $30 \mathrm{~min}$, and the membrane was incubated with the secondary antibodies (Abcam) for an additional $1 \mathrm{~h}$ at room temperature. The membrane was washed three times with TBST for $30 \mathrm{~min}$ and visualized using the enhanced chemiluminescence Western blot detection system (EMD Millipore, Billerica, MA, USA). Reference gene for 
Table I. Primers used in the present study.

A, Primers used for plasmid construction

\begin{tabular}{ll}
\hline Primers & Sequence \\
\hline shRNA-PCSK9-1 & \\
Forward & 5'GATCCCCTCCACTTCTCTGCCAAAGATTCAAGAGATCTTTGGCAGAGAAGTGGATTTTTA3' \\
Reverse & 5'AGCTTAAAAATCCACTTCTCTGCCAAAGATCTCTTGAATCTTTGGCAGAGAAGTGGAGGG3' \\
shRNA-PCSK9-2 & \\
Forward & 5'GATCCCCCAGAGTGACCACCGGGAAATTCAAGAGATTTCCCGGTGGTCACTCTGTTTTTA3' \\
Reverse & 5'AGCTTAAAAACAGAGTGACCACCGGGAAATCTCTTGAATTTCCCGGTGGTCACTCTGGGG3'
\end{tabular}

B, Primers used for RT-qPCR

\begin{tabular}{ll} 
GAPDH & \\
Forward & 5'-CACATGGCCTCCAAGGAGTA-3' \\
Reverse & 5'-TCCCCTCTTCAAGGGGTCTA -3' \\
PCSK9 & \\
Forward & 5'-TGGAACTCACTCACTCTGGG-3' \\
Reverse & 5'-AAGAATCCTGCCTCCTTGGT-3' \\
Bax & \\
Forward & 5'-TGATCAGAACCATCATGGGC-3' \\
Reverse & 5'-GGACATCAGTCGCTTCAGTG-3' \\
Caspase 3 & \\
Forward & \\
Reverse & 5'-GAGGCCGACTTCTTGTATGC-3' \\
Bcl-2 & 5'-GTTTCAGCATGGCACAAAGC-3' \\
Forward & \\
Reverse & 5'-TGATGGGATCGTTGCCTTATG-3' \\
\hline
\end{tabular}

shRNA, short hairpin RNA; PCSK9, proprotein convertase subtilisin/kexin type 9; RT-qPCR, reverse transcription-quantitative polymerase chain reaction; Bax, bcl-2-like protein 4; Bcl-2, B-cell lymphoma 2.

Table II. Antibodies used in the present study.

\begin{tabular}{|c|c|c|c|c|c|c|c|c|}
\hline \multirow[b]{2}{*}{ Antibody } & \multirow[b]{2}{*}{ Supplier } & \multicolumn{2}{|c|}{ Primary antibody } & \multicolumn{4}{|c|}{ Secondary antibody } & \multirow[b]{2}{*}{$\begin{array}{c}\text { Molecular } \\
\text { weight }(\mathrm{kD})\end{array}$} \\
\hline & & $\begin{array}{l}\text { Catalog } \\
\text { number }\end{array}$ & Dilution & $\begin{array}{c}\text { Species } \\
\text { raised }\end{array}$ & Conjugation & $\begin{array}{l}\text { Catalog } \\
\text { number }\end{array}$ & Dilution & \\
\hline PCSK9 & Sigma; Merck KGaA & SAB1302902 & $1: 500$ & Rabbit & HRP & ab191866 & $1: 2,000$ & 72 \\
\hline Caspase-3 & Abcam & ab32042 & $1: 100$ & Rabbit & HRP & ab191866 & $1: 5,000$ & 32 \\
\hline Bax & Abcam & ab32503 & $1: 5,000$ & Rabbit & HRP & ab191866 & $1: 10,000$ & 21 \\
\hline $\mathrm{Bcl}-2$ & Abcam & ab32124 & $1: 1,000$ & Rabbit & HRP & ab191866 & $1: 5,000$ & 26 \\
\hline p38 & Abcam & ab27986 & $1: 1,000$ & Rabbit & HRP & ab191866 & $1: 10,000$ & 38 \\
\hline p-p38 & Abcam & ab4822 & $1: 1,000$ & Rabbit & HRP & ab191866 & $1: 10,000$ & 38 \\
\hline ERK & Abcam & ab17942 & $1: 1,000$ & Rabbit & HRP & ab191866 & $1: 5,000$ & $42-44$ \\
\hline p-ERK & Abcam & $a b 214362$ & $1: 1,000$ & Rabbit & HRP & ab191866 & $1: 5,000$ & $42-44$ \\
\hline JNK & Abcam & ab179461 & $1: 1,000$ & Rabbit & HRP & ab191866 & $1: 5,000$ & 46 \\
\hline p-JNK & Abcam & ab124956 & $1: 1,000$ & Rabbit & HRP & ab191866 & $1: 5,000$ & 46 \\
\hline$\beta$-actin & Abcam & ab8226 & $1: 10,000$ & Mouse & HRP & ab131368 & $1: 10,000$ & 43 \\
\hline
\end{tabular}

Sigma; Merck KGaA (Darmstadt, Germany) and Abcam (Cambridge, UK); PCSK9, proprotein convertase subtilisin/kexin type 9; Bax, bcl-2-like protein 4; Bcl-2, B-cell lymphoma 2; p-, phosphorylated; ERK, extracellular signal-regulated kinases; JNK, c-Jun N-terminal kinases; HRP, horseradish peroxidase. 
A
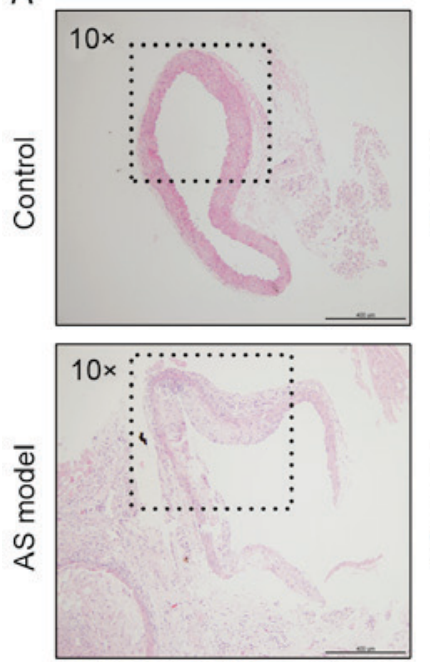
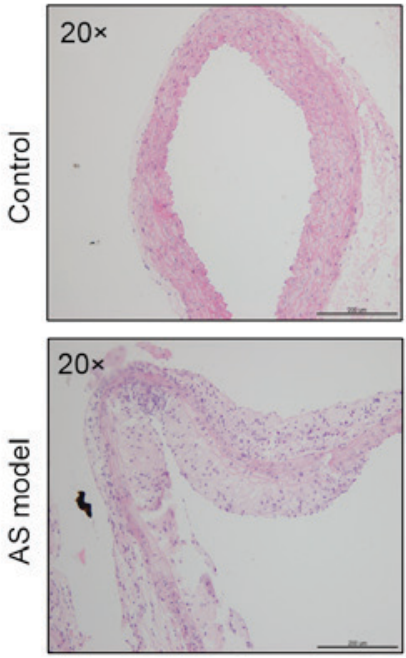

B
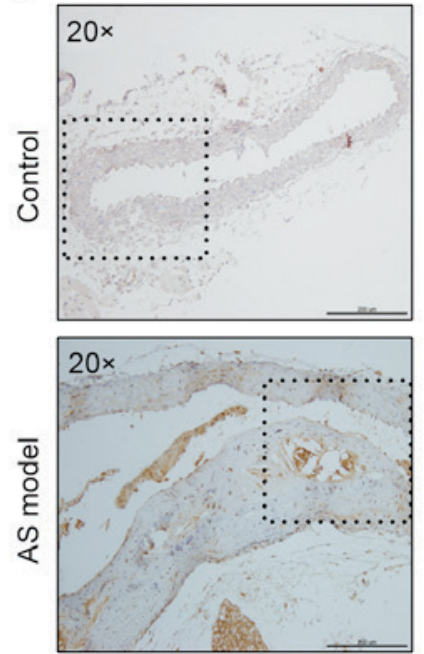
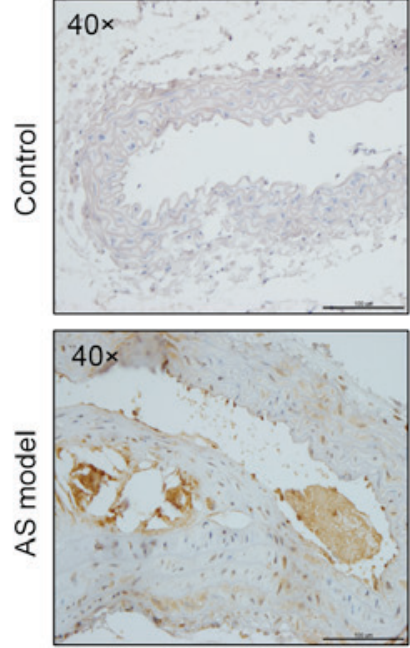

Figure 1. Expression of PCSK9 in AS. (A) AS mouse model. Hematoxylin-eosin staining of the cross section of aorta demonstrated that the AS mouse model was successfully developed in the ApoE ${ }^{-/-}$mice. (B) PCSK9 protein in AS mouse model was tested by immunohistochemical analysis. PCSK9, proprotein convertase subtilisin/kexin type 9; AS, atherosclerosis; ApoE, apolipoprotein E.

the western blot analysis was $\beta$-actin. All antibody details are listed in Table II.

Immunohistochemical analysis. Dissected mouse aortae were fixed in $4 \%$ polyformaldehyde (room temperature for $24 \mathrm{~h}$ ), embedded in paraffin wax and cut into sections of 3-5 $\mu \mathrm{m}$. A total of 5 sections were placed on each slide and the slides placed in an oven at $60^{\circ} \mathrm{C}$ for $2 \mathrm{~h}$. Following dewaxing by xylene, the sections were passed through antigen hot fix $\left(121^{\circ} \mathrm{C}\right.$ for $\left.10 \mathrm{~min}\right), \mathrm{DAB}$ staining and hematoxylin staining. The anti-PCSK 9 antibody was used at a dilution of 1:200 $\left(4^{\circ} \mathrm{C}\right.$ overnight). Finally, the sections were mounted with neutral gum. The expression of PCSK9 in the tissues was observed under an optical microscope.

Statistical analysis. All the data are expressed as the mean \pm standard error of the mean. The statistical difference between two experimental groups was determined using the Student's t-test and SPSS software (version 19.0; IBM SPSS, Armonk, NY, USA). $\mathrm{P}<0.05$ was considered to indicate a statistically significant difference.

\section{Results}

PCSK9 is highly expressed in atherosclerotic plaques. As demonstrated in Fig. 1A, the aortic structure and the vascular endothelium of the control group was complete and the elastic plate was evident. Notably, there were no plaques in the arteries. However, the aorta of the AS model group exhibited marked plaque formation and lipid deposition. In addition, the vascular endothelial was incomplete, the smooth muscle layer was broken and there was an infiltration of numerous inflammatory cells.

To determine the association between PCSK9 and AS, the aortae of the mice were analyzed by immunohistochemistry. The nuclei of aortae in the control group were stained clearly; however, there were no brown-yellow particles around the blue nucleus. This indicated that PCSK9 is barely expressed in normal blood vessels. By contrast, there were numerous
PCSK9 positive expression particles in the aortic plaque of the high-fat group. It demonstrated that PCSK9 exhibited an increased expression level in atherosclerotic plaques (Fig. 1B). The results suggest that the expression level of PCSK9 was closely associated with the presence of AS.

Ox-LDL induces the apoptosis of EAhy926 cells. Damage to endothelial cells is considered to be an important basis of the occurrence of AS. Endothelial cell apoptosis is able to alter the integrity of the endothelium, promote plaque formation and increase instability of plaques. Ox-LDL is a strong apoptosis-inducing factor in plaque lesions, and is able to induce apoptosis in a number of ways. EAhy926 cells were treated with ox-LDL (50 and $100 \mu \mathrm{g} / \mathrm{ml}$ ) at different time points. Cells were stained with an Annexin V FITC apoptosis kit and the apoptotic rate was detected by flow cytometry. The results demonstrated that ox-LDL is able to induce apoptosis in endothelial cells. The apoptotic rates for $50 \mu \mathrm{g} / \mathrm{ml}$ at 0,12 , 24,36 and $48 \mathrm{~h}$ were 14.36, 14.13, 22.48, 16.6 and 19.34\%, respectively. The apoptotic rate of the $100 \mu \mathrm{g} / \mathrm{ml}$ group were 14.36, 14.33, 24.19, 15.69 and $14.21 \%$. They demonstrated the highest apoptotic rate resulting from $24 \mathrm{~h}$ treatment. However, no significant difference between the two groups was observed (Fig. 2). Therefore, $50 \mu \mathrm{g} / \mathrm{ml}$ ox-LDL was used in the subsequent experiments.

Ox-LDL upregulates pro-apoptotic factors Bax and caspase-3, and downregulates anti-apoptotic factor Bcl-2 expression. Bax in the Bcl-2 family and caspase-3 in the caspase-family serve a key role in the promotion of apoptosis. Bcl-2 is also the main factor in the anti-apoptosis process. In the present study, HUVECs were treated with $50 \mu \mathrm{g} / \mathrm{ml}$ ox-LDL at different time points. The levels of Bax, Bcl-2 and caspase-3 mRNA and protein were measured using RT-qPCR and western blot analysis, respectively. With the increase of processing time, the expression of Bax (Fig. 3A) and caspase-3 (Fig. 3B) mRNA presented a trend of first increase and then decrease, with the highest expression of resulting from $24 \mathrm{~h}$ treatments (Bax: $\mathrm{P}<0.05$; caspase- 3 : 


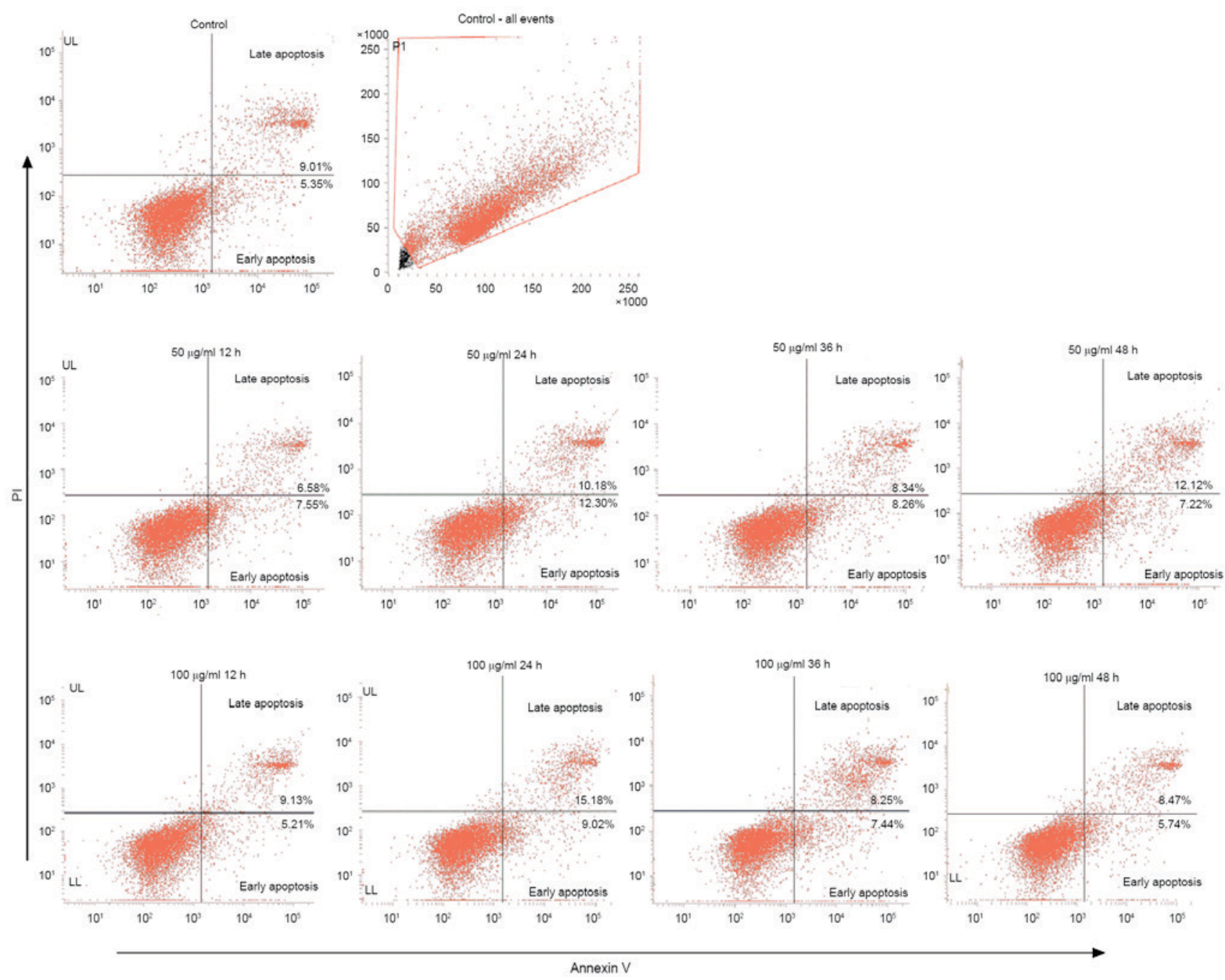

Figure 2. Apoptosis of human umbilical vein endothelial cells induced by oxidized low-density lipoprotein. PI, propidium iodide.

$\mathrm{P}<0.05)$. The expression levels of Bax and caspase-3 protein were upregulated in a time-dependent manner (Fig. 3C and D). By contrast, Bcl-2 was decreased in mRNA $(\mathrm{P}<0.05$; Fig. $3 \mathrm{E})$ and protein (Fig. 3F) levels induced by ox-LDL.

Ox-LDL upregulates PCSK9 expression in EAhy926 cells. Although PCSK9 is highly expressed in the AS plaques, the present study investigated whether it is also expressed in endothelial cells and whether it is involved in the apoptosis of endothelial cells. EAhy926 cells were treated with $50 \mu \mathrm{g} / \mathrm{ml}$ ox-LDL at different time periods and the levels of PCSK9 mRNA were measured using RT-qPCR. As demonstrated in Fig. 3G, the amounts of PCSK9 mRNA exhibited a gradually increased trend, and reached a peak at $24 \mathrm{~h}(\mathrm{P}<0.001)$. The amounts of PCSK9 protein were determined by western blot analysis. Ox-LDL increased the amounts of PCSK9 in a time-dependent manner(Fig. $3 \mathrm{H})$. These results suggested that ox-LDL upregulated the mRNA expression and protein level of PCSK9 during endothelial cell apoptosis, implying that PCSK9 may be involved in the development of AS by participating in the apoptosis of endothelial cells.

Downregulation of PCSK9 by shRNA-PCSK9 reduces the apoptotic rate of EAhy926 cells. Following transfected by
shRNA, the cells were treated with $50 \mu \mathrm{g} / \mathrm{ml}$ ox-LDL for $24 \mathrm{~h}$ and the apoptotic rate was detected. The apoptotic rate of 279 vector, shRNA-PCSK9-1 and shRNA-PCSK9-2 were 20.17, 12.72 and $10.95 \%$, respectively (Fig. 4). The results demonstrated that the apoptosis of EAhy926 cells is reduced with downregulation of PCSK9.

Detection of transfection efficiency. To downregulate PCSK9, EAhy926 cells were transfected with shRNA-PCSK9 packaged by lentivirus. PCSK 9 mRNA and protein levels were detected in the ox-LDL treated endothelial cells. As demonstrated in Fig. 5A, compared with the slow virus without the target plasmid (279-vector), the PCSK9 level was significantly reduced by transfected shRNA-PCSK9-1 and shRNA-PCSK9-2 ( $\mathrm{P}<0.001$; Fig. 5A).

Downregulation of PCSK9 by shRNA-PCSK9 affects apoptosis related proteins and mitogen-activated protein kinase (MAPK) pathway proteins expression induced by ox-LDL. Compared with the control group (279-vector), levels of pro-apoptotic proteins, including Bax and caspase-3, were suppressed in EAhy926 cells harboring shRNA-PCSK9, while anti-apoptosis protein $\mathrm{Bcl}-2$ was increased in mRNA and protein levels (Fig. 5B and C). 

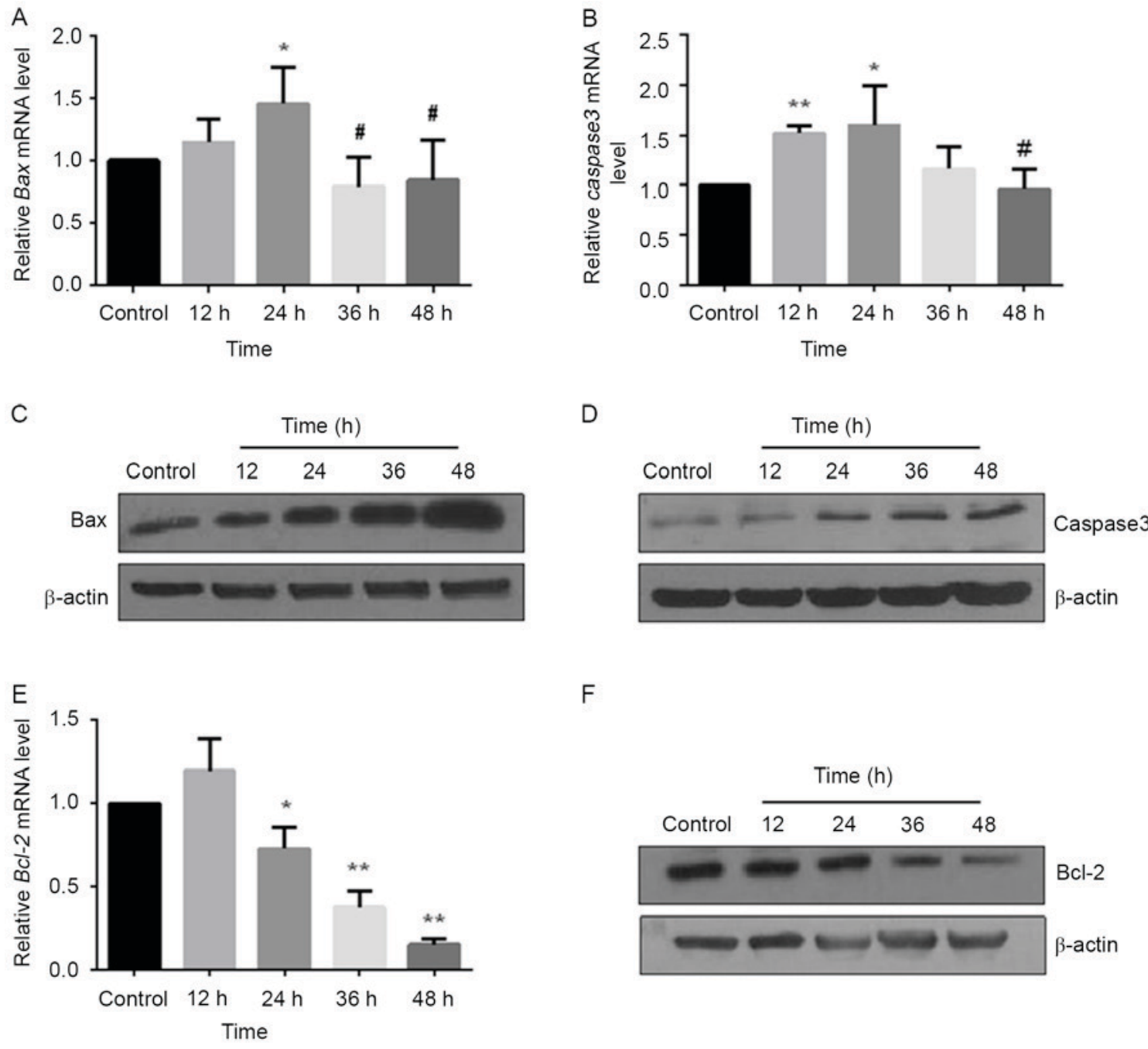

$\mathrm{F}$

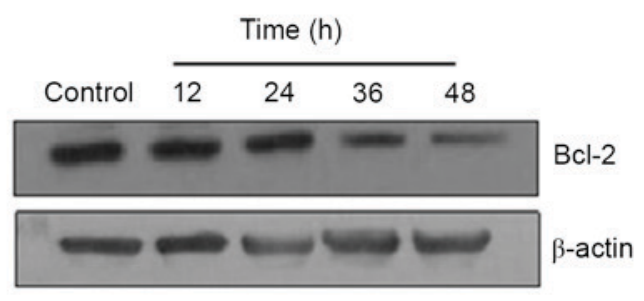

G

$\mathrm{H}$
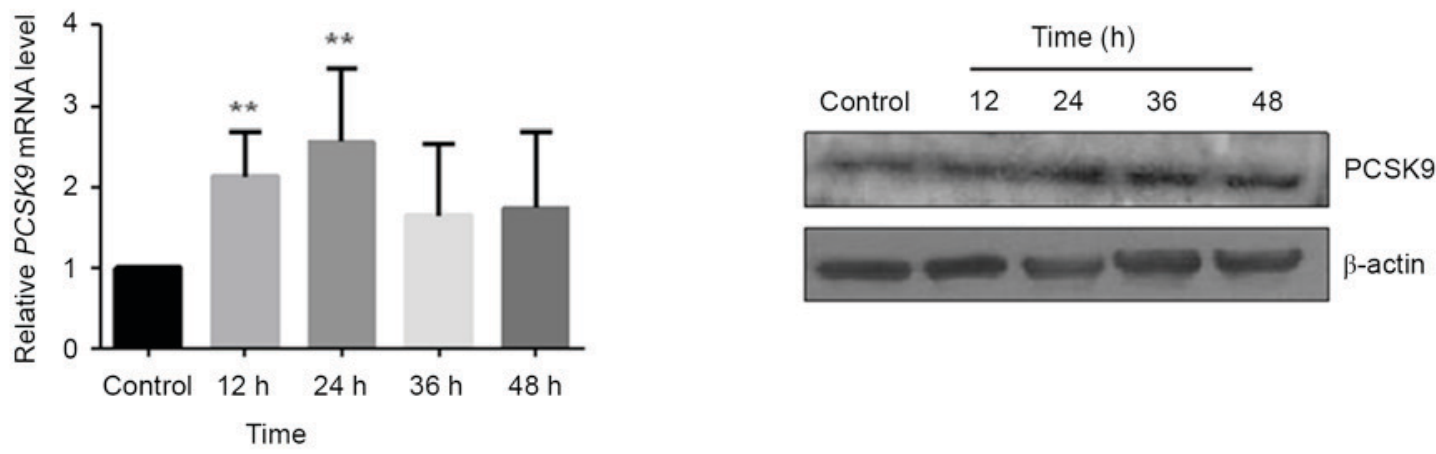

Figure 3. Effects of ox-LDL on Bax, Bcl-2, caspase-3 and PCSK9 expression levels in HUVECs at different times. EAhy926 cells were induced by ox-LDL at $50 \mu \mathrm{g} / \mathrm{ml}$. The mRNA levels of (A) Bax and (B) caspase-3 were detected by RT-qPCR. The protein levels of (C) Bax and (D) caspase-3 were detected by western blotting. Bcl-2 (E) mRNA and (F) protein levels were evaluated. PCSK9 (G) mRNA and (H) protein levels were evaluated. ${ }^{*} \mathrm{P}<0.05$ and ${ }^{* *} \mathrm{P}<0.01$ vs. control; " $\mathrm{P}<0.05$ vs. 24 h. All results are expressed as the mean \pm standard error of the mean. Ox-LDL, oxidized low-density lipoprotein; Bax, bcl-2-like protein 4; Bcl-2, B-cell lymphoma 2; PCSK9, proprotein convertase subtilisin/kexin type 9; HUVECs, human umbilical vein endothelial cells; RT-qPCR, reverse transcription-quantitative polymerase chain reaction.

Considering that MAPK signaling has been implicated in cells apoptosis, it was subsequently assessed whether the pathway was involved in the PCSK9-induced endothelial apoptotic response in AS. No significant effects of shRNA-PCSK9 on protein expression levels of JNK and ERK were determined. However, p38, phosphorylation of p38 and phosphorylation of JNK were inhibited (Fig. 5C).

\section{Discussion}

AS is the primary cause of mortality in developed countries and a number of developing countries, and is characterized by plaque formation (15). The rupture of an AS plaque may cause acute coronary syndrome, a threat to human health (16). Although there are a number of factors in association with 

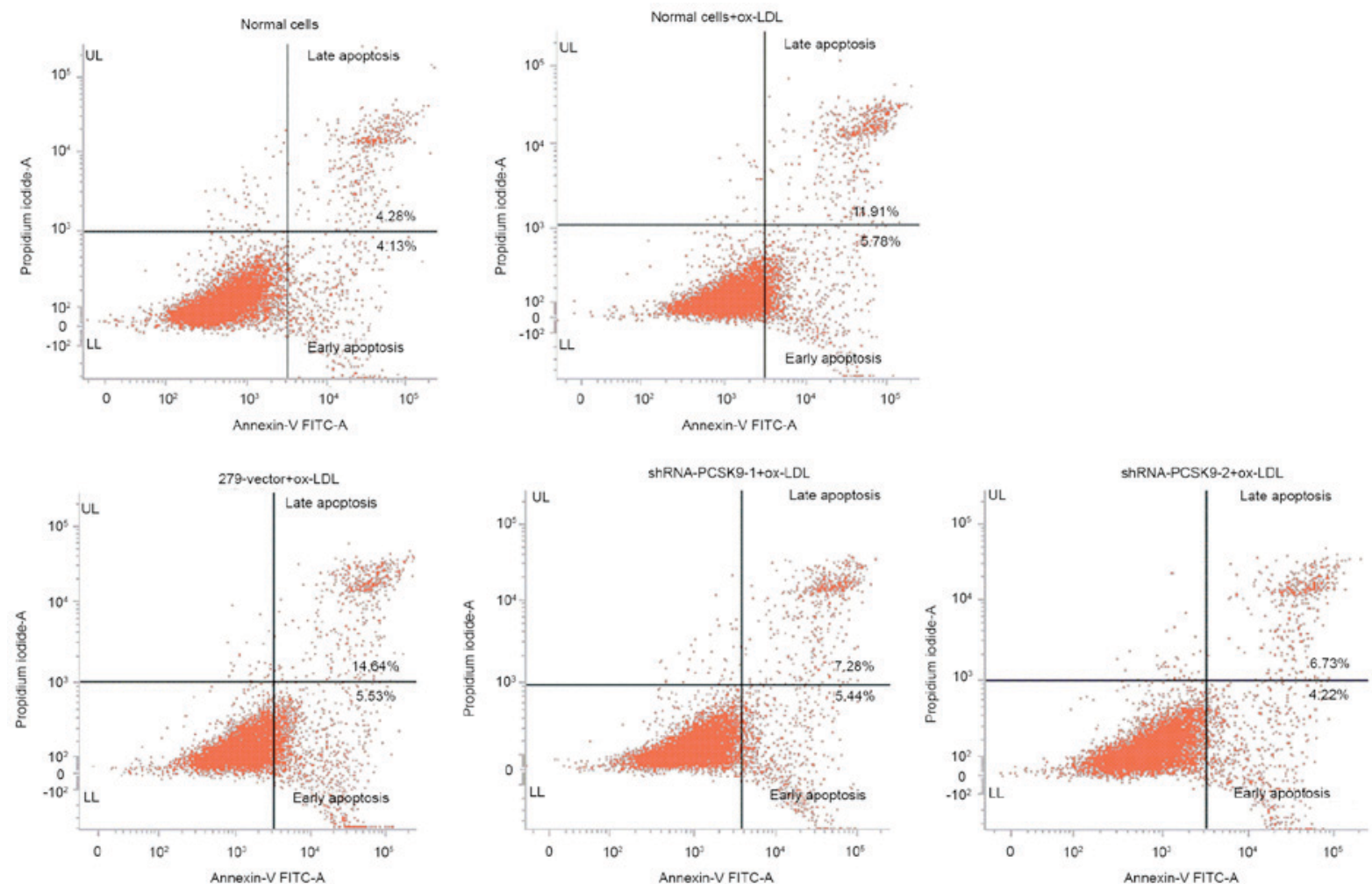

Figure 4. Apoptosis of human umbilical vein endothelial cells induced by ox-LDL following downregulation of PCSK9. ox-LDL, oxidized low-density lipoprotein; PCSK9, proprotein convertase subtilisin/kexin type 9; shRNA, short hairpin RNA.

A
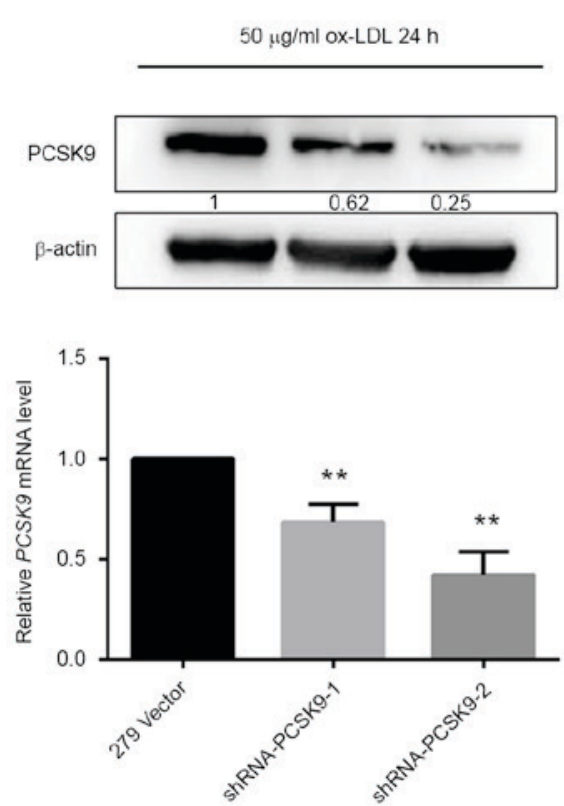

B

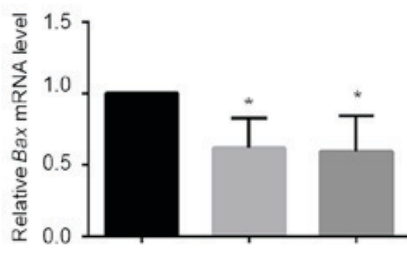

$\bar{\Phi}$

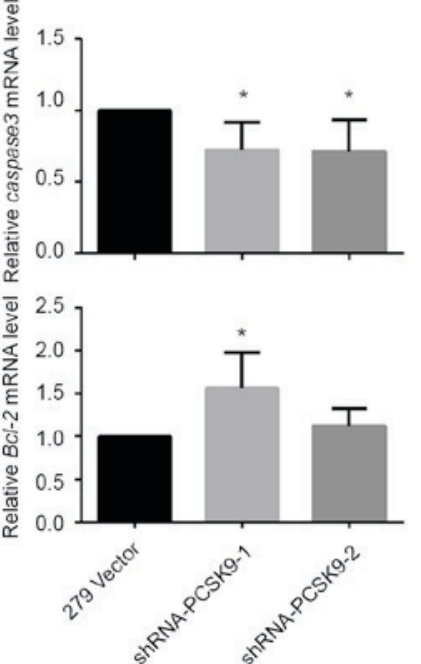

C
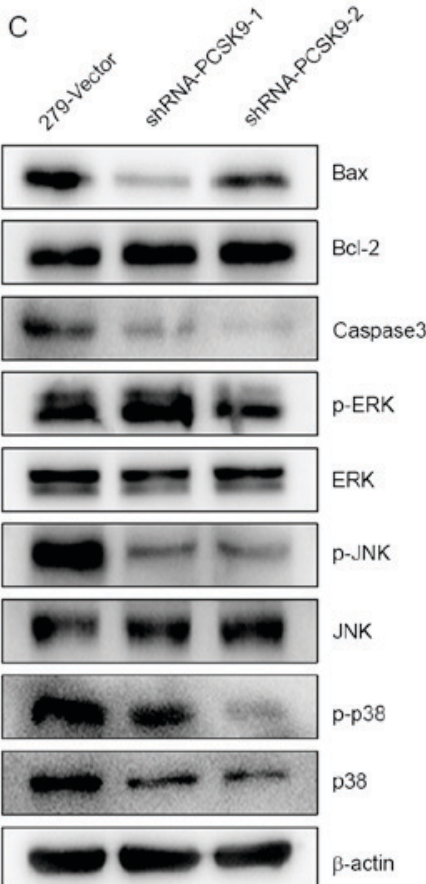

Figure 5. Effects of shRNA-PCSK9 on expression of apoptosis-associated and MAPK pathway proteins. EAhy926 cells were transfected with PCSK9 shRNA and induced by $50 \mu \mathrm{g} / \mathrm{ml}$ ox-LDL over $24 \mathrm{~h}$. (A) Effects of shRNA-PCSK9 on PCSK9 mRNA and protein levels. (B) Effects of PCSK9 deficiency on Bax, Bcl-2 and caspase-3 mRNA expression levels. (C) Effects of PCSK9 deficiency on Bax, Bcl-2, caspase-3, p38, ERK and JNK protein expression levels and phosphorylation in the ox-LDL treated EAhy 926 cells. ${ }^{*} \mathrm{P}<0.05$ and ${ }^{* *} \mathrm{P}<0.01$ vs. 279 -vector. All results are expressed as the mean \pm standard error of the mean. shRNA, short hairpin RNA; PCSK9, proprotein convertase subtilisin/kexin type 9; MAPK, mitogen-activated protein kinase; ox-LDL, oxidized low-density lipoprotein; Bax, bcl-2-like protein 4; Bcl-2, B-cell lymphoma 2; ERK, extracellular signal-regulated kinases; JNK, c-Jun N-terminal kinases; p-, phosphorylated. 
AS, including age, sex, smoking, diabetes, hypertension and hyperlipidemia, the initiating factors remain to be completely elucidated (17). Substantial evidence suggests that AS is a chronic inflammatory and multifactorial disease, in which vascular endothelial cell dysfunction serves an important role (18). Vascular endothelial cells are located on the inner surface of the vessel wall, which acts as an important permeability barrier between circulating blood and tissue. These cells are also involved in cellular cholesterol, lipid homeostasis, signal transduction, inflammation and immunity (19). Excessive apoptosis of endothelial cells promotes the formation and rupture of unstable plaques, which cause arterial wall abnormalities in morphogenesis, stable structure and metabolism. Therefore, the role of cell apoptosis cannot be ignored (20).

In previous years, increasing attention has been focused on PCSK9, a newly discovered gene associated with autosomal dominant hypercholesterolemia. Similar to LDLR and apolipoprotein (Apo) B-100, PCSK9 serves an important role in lipid metabolism. Gene mutations or polymorphisms of PCSK9 are involved in familial hypercholesterolemia and lead to a high risk of atherosclerotic cardiovascular disease (21-23). The removal of LDL-C depends on the binding efficiency of LDL particles and LDLR. Following the clearing of LDL-C, LDLR is released and recycled to the liver cell surface, thus LDLR serves a crucial role in cholesterol homeostasis. A number of studies (24-26) have confirmed that PCSK9 is able to reduce the amount of LDLR and interfere with the LDL-C removal process. PCSK9 is secreted into the blood by hepatocytes and subsequently binds to LDLR, becoming internalized into the lysosome for degradation (27). Based on this, PCSK9 is able to regulate the plasma concentration of LDL-C beyond the cellular level. However, the effect of PCSK9 on the degradation of LDLR is independent of its catalytic activity; however, in its role as a molecular chaperone of LDLR recycling (28). According to the present study, PCSK9 is also able to exhibit a direct toxic effect on the vascular wall.

Apo is a protein component of plasma lipoproteins and is divided into five groups: A, B, C, D and E. ApoE removes ligands, participating in a receptor-mediated apo-lipoprotein cleaning process, primarily formed of chylomicrons and very LDLs (29). ApoE gene knockout may lead to upregulation of total plasma cholesterol and LDL-C, thus inducing arterial intimal injury and lipid deposition, and ultimately the development of AS (30). Therefore, the present study used $\mathrm{ApoE}^{-/-}$mice on a high-fat diet, successfully establishing an AS model. Immunohistochemical analysis of mice aortae demonstrated that PCSK9 was highly expressed in AS plaques and the expression levels of PCSK9 are associated with AS.

RNA interference (RNAi) is a highly conserved and post-transcriptional gene silencing phenomenon, induced by a double stranded RNA molecule that triggers the highly efficient degradation of homologous mRNA. It is widespread in the evolutionary process. At the beginning of the 1990s, the botanist first identified this phenomenon and designated it gene repression (31). Fire et al (32) also identified the inhibition of endogenous gene expression caused by this double stranded RNA in a nematode and was the first to designate it RNAi. In 2001, RNAi was observed in mammalian cells (33). RNAi was gradually demonstrated to exist widely in a number of eukaryotic organisms.
The apoptosis of vascular endothelial cells is an important factor in the pathogenesis of AS and is an important target for preventing and delaying the disease (34). The Bcl protein family is an important factor in apoptosis regulation, in which $\mathrm{Bcl}-2$ is an anti-apoptotic protein and Bax is a pro-apoptotic protein. The shift in the balance between pro-apoptotic and anti-apoptotic proteins leads to the occurrence of apoptosis (35). In the process of inducing apoptosis, the activation of the caspase pathway is another specific marker (36). Caspase is frequently used as a target for anti-AS drugs in the process of endothelial cell apoptosis and the development of AS (37). In the present study, apoptosis was induced in HUVECs by ox-LDL and the expression of pro-apoptotic protein Bax and caspase-3 was significantly promoted in a time-dependent manner. Conversely, expression of Bcl-2 was blocked. At the same time, PCSK9 expression in mRNA and protein levels was significantly increased in apoptotic HUVECs compared with normal cells. However, PCSK9 was downregulated during shRNA-PCSK9 transfection. The present study also identified that transfection of cells with shRNA-PCSK9 inhibited the mRNA and protein expression of Bax and caspase-3, while $\mathrm{Bcl}-2$ demonstrated the opposite role. The ratio of $\mathrm{Bcl}-2 / \mathrm{Bax}$ was increased by PCSK9 deficiency in ox-LDL-induced apoptosis.

The MAPK signaling pathway is one of the most important in the eukaryotic signaling network, involved in a variety of cellular functions, particularly cell proliferation, differentiation and apoptosis (38). It has been reported that JNK and 38 MAPK serve an important role in inflammation and cell apoptosis due to activation by TNF- $\alpha$, IL-1, G protein coupled receptors, stress and oxidative damage (39). The ERK signaling pathway serves an important role in the process of cell proliferation mediated by growth factors (40). It is notable that the sustained activation of ERK can prevent the occurrence of apoptosis (41). In the present study, shRNA-PCSK9 markedly decreased the phosphorylation of p38 and JNK, suggesting that the p38/JNK-dependent pathway may be involved in PCSK9-mediated endothelial cell apoptosis in AS.

In conclusion, the findings of the present study suggested that PCSK9 promotes the apoptosis of endothelial cells induced by ox-LDL in AS via the JNK/p38 MAPK pathway. The comprehensive understanding of PCSK9 provides a new insight into the mechanism of AS and a new target and direction for its treatment.

\section{Acknowledgements}

The present study was supported by grants to G.L. from the National Natural Science Foundation of China (grant no. 81370300) and the Specialized Research Fund for the Doctoral Program of Higher Education (grant no. 20121202110004), and a grant to Y.W. from the Science and Technology Foundation of Tianjin Sanitary Bureau (grant no. 2015KZ105).

\section{References}

1. Pescetelli I, Zimarino M, Ghirarduzzi A and De Caterina R: Localizing factors in atherosclerosis. J Cardiovasc Med (Hagerstown) 16: 824-830, 2015. 
2. Yusuf S, Hawken S, Ounpuu S, Dans T, Avezum A, Lanas F, McQueen M, Budaj A, Pais P, Varigos J, et al: Effect of potentially modifiable risk factors associated with myocardial infarction in 52 countries (the INTERHEART study): Case-control study. Lancet 364: 937-952, 2004

3. Weber $\mathrm{C}$ and Noels H: Atherosclerosis: Current pathogenesis and therapeutic options. Nat Med 17: 1410-1422, 2011.

4. Napoli C: Oxidation of LDL, atherogenesis, and apoptosis. Ann N Y Acad Sci 1010: 698-709, 2003.

5. Lu J, Mitra S, Wang X, Khaidakov M and Mehta JL: Oxidative stress and lectin-like ox-LDL-receptor LOX-1 in atherogenesis and tumorigenesis. Antioxid Redox Signal 15: 2301-2333, 2011.

6. Geng YJ: Biologic effect and molecular regulation of vascular apoptosis in atherosclerosis. Curr Atheroscler Rep 3: 234-242, 2001.

7. Cai $\mathrm{H}$ and Harrison DG: Endothelial dysfunction in cardiovascular diseases: The role of oxidant stress. Circ Res 87: 840-844, 2000.

8. Abifadel M, Guerin M, Benjannet S, Rabès JP, Le Goff W, Julia Z, Hamelin J, Carreau V, Varret M, Bruckert E, et al: Identification and characterization of new gain-of-function mutations in the PCSK9 gene responsible for autosomal dominant hypercholesterolemia. Atherosclerosis 223: 394-400, 2012.

9. Roth EM, McKenney JM, Hanotin C, Asset G and Stein EA Atorvastatin with or without an antibody to PCSK9 in primary hypercholesterolemia. N Engl J Med 367: 1891-1900, 2012.

10. Lee P and Hegele RA: Current Phase II proprotein convertase subtilisin/kexin 9 inhibitor therapies for dyslipidemia. Expert Opin Investig Drugs 22: 1411-1423, 2013.

11. Poirier S and Mayer G: The biology of PCSK9 from the endoplasmic reticulum to lysosomes: New and emerging therapeutics to control low-density lipoprotein cholesterol. Drug Des Devel Ther 7: 1135-1148, 2013.

12. Leren TP: Sorting an LDL receptor with bound PCSK9 to intracellular degradation. Atherosclerosis 237: 76-81, 2014

13. Constantinides A, Kappelle PJ, Lambert G and Dullaart RP Plasma lipoprotein-associated phospholipase A2 is inversely correlated with proprotein convertase subtilisin-kexin type 9 . Arch Med Res 43: 11-14, 2012

14. Livak KJ and Schmittgen TD: Analysis of relative gene expression data using real-time quantitative PCR and the 2(-Delta Delta C(T)) method. Methods 25: 402-408, 2001.

15. Libby P, Ridker PM and Maseri A: Inflammation and atherosclerosis. Circulation 105: 1135-1143. 2002.

16. Rioufol G, Finet G, Ginon I, André-Fouët X, Rossi R, Vialle E, Desjoyaux E, Convert G, Huret JF and Tabib A: Multiple atherosclerotic plaque rupture in acute coronary syndrome: A three-vessel intravascular ultrasound study. Circulation 106 : 804-808, 2002

17. Gu HF, Tang CK and Yang YZ: Psychological stress, immune response, and atherosclerosis. Atherosclerosis 223: 69-77, 2012.

18. Hansson GK: Inflammation, atherosclerosis, and coronary artery disease. N Engl J Med 352: 1685-1695, 2005.

19. Simionescu M and Antohe F: Functional ultrastructure of the vascular endothelium: Changes in various pathologies. Handb Exp Pharmacol: 41-69, 2006.

20. Kwon GP, Schroeder JL, Amar MJ, Remaley AT and Balaban RS: Contribution of macromolecular structure to the retention of low-density lipoprotein at arterial branch points. Circulation 117: 2919-2927, 2008

21. Benn M, Nordestgaard BG, Grande P, Schnohr P and TybjaergHansen A: PCSK9 R46L, low-density lipoprotein cholestero levels, and risk of ischemic heart disease: 3 independent studies and meta-analyses. J Am Coll Cardiol 55: 2833-2842, 2010.
22. Zhang L, Yuan F, Liu P, Fei L, Huang Y, Xu L, Hao L, Qiu X, Le Y, Yang X, et al: Association between PCSK9 and LDLR gene polymorphisms with coronary heart disease: Case-control study and meta-analysis. Clin Biochem 46: 727-732, 2013.

23. Wu NQ and Li JJ: PCSK9 gene mutations and low-density lipoprotein cholesterol. Clin Chim Acta 431: 148-153, 2014.

24. Wang Y,Huang Y,Hobbs HH and Cohen JC: Molecular characterization of proprotein convertase subtilisin/kexin type 9-mediated degradation of the LDLR. J Lipid Res 53: 1932-1943, 2012.

25. Tavori H, Fan D, Blakemore JL, Yancey PG, Ding L, Linton MF and Fazio S: Serum proprotein convertase subtilisin/kexin type 9 and cell surface low-density lipoprotein receptor: Evidence for a reciprocal regulation. Circulation 127: 2403-2413, 2013.

26. Nguyen MA, Kosenko T and Lagace TA: Internalized PCSK 9 dissociates from recycling LDL receptors in PCSK9-resistant SV-589 fibroblasts. J Lipid Res 55: 266-275, 2014.

27. Zhang DW, Lagace TA, Garuti R, Zhao Z, McDonald M, Horton JD, Cohen JC and Hobbs HH: Binding of proprotein convertase subtilisin/kexin type 9 to epidermal growth factor-like repeat A of low density lipoprotein receptor decreases receptor recycling and increases degradation. J Biol Chem 282: 18602-18612, 2007.

28. Mousavi SA, Berge KE and Leren TP: The unique role of proprotein convertase subtilisin/kexin 9 in cholesterol homeostasis. J Intern Med 266: 507-519, 2009.

29. Plump AS and Breslow JL: Apolipoprotein E and the apolipoprotein E-deficient mouse. Annu Rev Nutr 15: 495-518, 1995.

30. Getz GS and Reardon CA: Animal models of atherosclerosis. Arterioscler Thromb Vasc Biol 32: 1104-1115, 2012.

31. Jorgensen RA: Cosuppression, flower color patterns, and metastable gene expression states. Science 268: 686-691, 1995.

32. Fire A, Xu S, Montgomery MK, Kostas SA, Driver SE and Mello CC: Potent and specific genetic interference by double-stranded RNA in Caenorhabditis elegans. Nature 391: 806-811, 1998

33. Elbashir SM, Martinez J, Patkaniowska A, Lendeckel W and Tuschl T: Functional anatomy of siRNAs for mediating efficient RNAi in Drosophila melanogaster embryo lysate. EMBO J 20: 6877-6888, 2001

34. Onat D, Brillon D, Colombo PC and Schmidt AM: Human vascular endothelial cells: A model system for studying vascular inflammation in diabetes and atherosclerosis. Curr Diab Rep 11: 193-202, 2011.

35. Berens HM and Tyler KL: The proapoptotic Bcl-2 protein Bax plays an important role in the pathogenesis of reovirus encephalitis. J Virol 85: 3858-3871, 2011.

36. Sanz AB, Santamaría B, Ruiz-Ortega M, Egido J and Ortiz A: Mechanisms of renal apoptosis in health and disease. J Am Soc Nephrol 19: 1634-1642,2008.

37. Zhang Y, Chen N, Zhang J and Tong Y: Hsa-let-7g miRNA targets caspase-3 and inhibits the apoptosis induced by ox-LDL in endothelial cells. Int J Mol Sci 14: 22708-22720, 2013.

38. Aplin AE, Hogan BP, Tomeu J and Juliano RL: Cell adhesion differentially regulates the nucleocytoplasmic distribution of active MAP kinases. J Cell Sci 115: 2781-2790, 2002.

39. Weston CR and Davis RJ: The JNK signal transduction pathway. Curr Opin Genet Dev 12: 14-21, 2002.

40. Poulikakos PI, Persaud Y, Janakiraman M, Kong X, Ng C, Moriceau G, Shi H, Atefi M, Titz B, Gabay MT, et al: RAF inhibitor resistance is mediated by dimerization of aberrantly spliced BRAF(V600E). Nature 480: 387-390, 2011.

41. Krens SF, Spaink HP and Snaar-Jagalska BE: Functions of the MAPK family in vertebrate-development. FEBS Lett 580: 4984-4990, 2006. 